Didáctica. Lengua y literatura

ISSN: 1130-0531

http://dx.doi.org/10.5209/DIDA.61953

\title{
Trabajar desde la memoria y desterrar lecto-mitos: Dos estrategias en la formación de lectores competentes de textos académicos ${ }^{1}$
}

\author{
Zahyra Camargo Martínez; Graciela Uribe Álvarez³ Miguel Ángel Caro Lopera ${ }^{4}$
}

Recibido: 27 de octubre de 2016 / Aceptado: 15 de mayo de 2018

Resumen. Este artículo es resultado directo del proyecto que investigadores del grupo DiLeMa (Didáctica de la Lengua Materna y la Literatura) adelantaron en el marco de un programa b-learning de intervención didáctica dirigido a docentes en formación y en ejercicio asociados a la Licenciatura en Español y Literatura, y a la Maestría en Ciencias de la Educación en la Universidad del Quindío (20142015). Se describen aquí dos estrategias que, en concepto del equipo investigador, podrían despertar la formación de lectores competentes de textos académicos: el estudio de la memoria como precursora de todo proceso de comprensión y la identificación de lecto-mitos como distractores de la acción didáctica. La conclusión defiende la idea de que el maestro como lector de su clase debe desarrollar una conciencia constructivo-integrativa derivada del modelo teórico interactivo propuesto por Teun van Dijk y Walter Kintsch.

Palabras clave: Comprensión lectora; didáctica; estrategias cognitivas y metacognitivas; textos académicos; Web 2.0.

\section{[en] Working From Memory and Banishing Reading-Myths: Two Strategies to Train Competent Readers of Academic Texts}

\footnotetext{
1 Este artículo deviene del proyecto Estrategias para lograr lectores competentes de textos académicos. Un programa interactivo de intervención didáctica dirigido a profesores de Educación Básica en el departamento del Quindio, realizado por el grupo de investigación DiLeMa (Didáctica de la Lengua Materna y la Literatura), Universidad del Quindío (2014-2015).

2 Universidad del Quindío (Colombia)

E-mail: zcamargo@uniquindio.edu.co

Doctora en Didáctica de las Lenguas y la Literatura de la Universidad Complutense de Madrid. Profesora titular del Programa de Español y Literatura, y de la Maestría y Doctorado en Ciencias de la Educación (Universidad del Quindío). Coordinadora del grupo de investigación DiLeMa (Didáctica de la Lengua Materna y la Literatura), Universidad del Quindío.

3 Universidad del Quindío (Colombia)

E-mail: guribe@uniquindio.edu.co

Doctora en Didáctica de las Lenguas y la Literatura de la Universidad Complutense de Madrid. Profesora titular del Programa de Español y Literatura y de la Maestría y Doctorado en Ciencias de la Educación (Universidad del Quindío). Investigadora del grupo DiLeMa (Didáctica de la Lengua Materna y la Literatura), Universidad del Quindío.

$4 \quad$ Universidad del Quindío (Colombia)

E-mail: macaro@uniquindio.edu.co

Magíster en Lingüística Lingüística (Universidad Tecnológica de Pereira). Profesor titular del Programa de Español y Literatura y de la Maestría en Ciencias de la Educación (Universidad del Quindío). Investigador del grupo DiLeMa (Didáctica de la Lengua Materna y la Literatura), Universidad del Quindío.
} 


\begin{abstract}
This article is a direct result of the project developed by researchers of the group DiLeMa (Didáctica de la Lengua Materna y la Literatura) framed within a didactic b-learning intervention program aimed at in training and in-service teachers belonging to the Spanish and Literature program and at the Master of Education Sciences at the University of Quindío (2014-2015). Here it is described two strategies that, according to the group's perspective, could awaken the training of competent readers of academic texts: the study of memory as a precursor of every comprehension process and the identification of "lecto-mitos" as distractors of the didactic action. The conclusion supports the idea that the teacher, as reader of his own class, must develop a constructivist-integrative awareness derived from an interactive theoretical model proposed by Teun van Dijk and Walter Kintsch.
\end{abstract}

Keywords: Reading comprehension; didactics; cognitive and metacognitive strategies; academic texts; Web 2.0.

\title{
[fr] Travailler à partir de la mémoire et bannir les lecto-mythes: deux stratégies dans la formation des lecteurs compétents des textes académiques
}

\begin{abstract}
Cet article est le résultat direct du projet que des enquêteurs du groupe DiLeMa (didactique de la langue maternelle et littérature) ont dirigé dans le cadre d'un programme blearning d'une intervention didactique qui s'adressait à des enseignants en formation et dans un exercice associé à la licence en espagnol et en littérature, et à la maîtrise en sciences de l'éducation à l'université du Quindío (20142015). On décrit ici deux stratégies qui, selon le concept de l'équipe investigatrice, pourraient éveiller la formation de lecteurs compétents de textes académiques : l'étude de la mémoire comme précurseure de tout processus de compréhension et l'identification de lectomythes qui dissipent l'action didactique. La conclusion défend l'idée que le maître en tant que «lecteur» de sa classe doit développer une conscience constructivointégrative dérivée du modèle théorique interactif proposé par Teun van Dijk et Walter Kintsch.
\end{abstract}

Keywords: Compréhension à la lecture ; didactique ; stratégies cognitives et métacognitives ; textes académiques ; Web 2.0.

Sumario. 1. Introducción. 2. Metodología. 3. Resultados y discusión. 3.1. El estudio de la memoria como precursora de todo proceso de comprensión de lectura. 3.2. La detección de lecto-mitos como distractores de la acción didáctica. 4. Conclusiones. 5. Bibliografía.

Cómo citar: Camargo Martínez, Z.; Uribe Álvarez, G.; Caro Lopera, M. Á. (2018) Trabajar desde la memoria y desterrar lecto-mitos: Dos estrategias en la formación de lectores competentes de textos académicos, Didáctica. Lengua y literatura, 30, 41-56.

\section{Introducción}

La reflexión sobre los lecto-mitos como distractores de aula se deriva de la investigación Estrategias para lograr lectores competentes de textos académicos. Un programa interactivo de intervención didáctica dirigido a profesores de Educación Básica en el Departamento del Quindío (Colombia), inscrita en la línea en Didáctica de la lengua materna y la literatura de la Licenciatura en Español y Literatura de la Universidad del Quindío. Parte del análisis del insuficiente desempeño en la comprensión global de los textos leídos que revelan los resultados obtenidos por los estudiantes en las pruebas de Logro SABER, en el Quindío ${ }^{5}$. Uno de los factores

$5 \quad$ Pruebas censales que se aplican a estudiantes de $5^{\circ}, 7^{\circ}$ y $9^{\circ}$ de Educación Básica en todo el país acerca de Lenguaje, Matemáticas y Ciencias Naturales. 
asociados a las falencias observadas es que en la escuela no se hace lo suficiente para desarrollar este tipo de competencias, debido a múltiples causas: 1) la frágil formación en los procesos de actualización de los profesores; 2) la falta de claridad de los mismos sobre los enfoques que orientan la práctica pedagógica; 3) el desconocimiento de modelos procesuales de comprensión lectora; y 4) la debilidad en las instituciones educativas para implementar sistemas de seguimiento y evaluación por competencias, que incluyan los requerimientos de los Lineamientos Curriculares y de los Estándares en el área de lenguaje.

Esta problemática plantea, entonces, la exigencia de pensar en nuevos enfoques, que permitan cambios significativos en los procesos de comprensión lectora de textos académicos, de manera que estos incidan efectivamente en el desarrollo cognitivo de los estudiantes y, por lo tanto, justifica la iniciación de procesos de formación y actualización de profesores, sustentados en experiencias investigativas y en proyectos de intervención didáctica, como los que se formulan desde esta investigación, que tiene sus bases en el hecho de que leer y escribir son esenciales para tener éxito en los ambientes educativos y en el trabajo. Esta investigación, por tanto, tiene implicaciones en la formación inicial y permanente del profesorado, ya que la capacitación didáctica del docente y la disponibilidad de materiales didácticos constituyen elementos esenciales para su actividad en el aula. Así, pues, la importancia de esta investigación radica en que, actualmente, la formación del profesorado solo contempla la enseñanza de la comprensión lectora en el área de lenguas, con lo cual, los profesores de las otras áreas del currículo no suelen recibir la preparación necesaria para introducir dentro del proceso de enseñanza y aprendizaje, la comprensión de textos académicos. Por esta razón, se desaprovecha una herramienta de aprendizaje eficaz, tanto en la adquisición de los conocimientos propios de los diversos espacios académicos, como en el perfeccionamiento de la competencia comunicativa.

En vista de la necesidad de formación de un significativo número de profesores, se propuso como objetivo general: Diseñar un programa interactivo de intervención didáctica para el desarrollo de la comprensión lectora mediante estrategias cognitivas y metacognitivas, dirigido a profesores de Educación Básica del Departamento del Quindío, con el fin de lograr lectores competentes de textos académicos. Como objetivos especificos se buscó:

1. Diseñar el programa interactivo en el ambiente de la Web 2.0.

2. Aplicar el programa interactivo inicialmente con estudiantes de la Licenciatura, futuros maestros y, posteriormente, con un grupo de docentes en ejercicio, mediante la metodología B-learning ${ }^{6}$.

3. Generar los materiales necesarios para el desarrollo de las estrategias cognitivas y metacognitivas.

4. Valorar los resultados de la intervención.

Las categorías que sustentan esta investigación se basan en los siguientes presupuestos teóricos: 1. Didáctica, que aborda las interacciones pedagógicas desde un punto de vista siempre orientado por los objetos de saber, y más precisamente por las condiciones de su transmisión y adquisición (Chevallard, 1991; Camps,

$6 \quad$ El b-learning (Blended Learning; en español, aprendizaje mezclado, semipresencial) es un método que combina prácticas virtuales y presenciales, al servicio de unas metas de aprendizaje. 
2004); 2. Competencias, relacionadas con la autonomía del aprendizaje en el nivel educativo, agrupadas en 6 bloques: Enseñar/aprender a aprender y pensar; enseñar/ aprender a cooperar; enseñar/aprender a comunicarse; enseñar/aprender a empatizar; enseñar/aprender a ser crítico y enseñar/aprender a automotivarse (Monereo y Pozo, 2003); 3. Competencia comunicativa, que exige un conocimiento, no solo lingüístico, textual, sociocultural y pragmático, sino también, procedimental, lo que permite potenciar el uso del lenguaje (Hymes, 1967 y 1995; Canale y Swain, 1980; Bachman, 1995); 4. Modelo constructivo-integrativo, que plantea un proceso secuenciado e interactivo, caracterizado por poner todo su énfasis en el análisis de la estructura del texto, la descripción formal semántica y el procesamiento psicológico. La comprensión lectora involucra, fundamentalmente, para estos autores, la construcción de una representación mental, de un modelo referencial o situacional de la realidad descrita por el texto (van Dijk y Kintsch, 1978 y 1983; Kintsch, 1998; Kintsch y Yarborough, 1982); 5. Texto, entendido como todo acto de habla, de cualquier extensión, que posea dimensión universal: hablante/oyente, medio de comunicación, discurso con forma y contenido, contexto y finalidad (Bronckart, 1996; Adam, 1992; Halliday, 1982); 6. Tipología expositivo-explicativa, definida como "todo texto cuyo objetivo principal es expresar información o ideas con la intención de mostrar y de explicar o hacer más comprensibles dichas informaciones" (Álvarez, 2001, 16); 7. Estrategias cognitivas, que exigen al lector procesar información, asimilar el texto, transformar el lenguaje y/o la representación del contenido, con el fin último de alcanzar el sentido y/o recordarlo; y las estrategias metacognitivas, que hacen referencia al conocimiento de los procesos y estrategias que el lector utiliza cuando se enfrenta a la comprensión, así como al control que pueda ejercer sobre procesos de lectura, con el fin de optimizarla (Gaskins y Elliot, 1999); 8. Web 2.0, reconocida como una de las tecnologías de información y comunicación que sirven como soporte técnico para provocar cambios en la didáctica de los procesos lectores y escritores, ya que permite el paso de una enseñanza pasiva a una colaborativa y cooperativa y pone al alcance del profesorado y del alumnado nuevas herramientas con un gran potencial didáctico.

Esta investigación se estructura, así, desde un enfoque cualitativo con el objetivo de solucionar un hecho problemático real; se orienta hacia el proceso y tiene en cuenta la necesidad de un abordaje teórico adecuado que permita a cada uno de los docentes planear, ejecutar y evaluar estrategias eficaces para sus entornos específicos de aula. El desarrollo del proyecto, en su dinámica metodológica, se llevó a cabo en tres fases: 1) Descriptiva y preparatoria: planificación; 2) Experimental: desarrollo de dos intervenciones: una con un grupo de docentes en formación, conformado por 20 estudiantes de la Licenciatura en Español y Literatura, futuros maestros; y otra con 15 profesores de Educación Básica, en ejercicio, adscritos a la Maestría en Ciencias de la Educación ${ }^{7}$, ambos programas de la Universidad del Quindío; 3) Análisis de resultados y conclusiones. Asimismo, con el fin de hacer un seguimiento al proceso de formación de profesores a través de la plataforma virtual, se diseñaron -además de los materiales centrales de lectura- actividades de monitoreo y control del proceso, tales como consultas, foros, pruebas, wikis, y se hizo uso de las listas de control e historiales propios de la web.

Vale la pena aclarar que esta maestría tiene énfasis en investigación y una duración prevista de dos años. 
Como resultados de esta experiencia interactiva, se resaltan 10 estrategias $^{8}$ que conjugan el modelo teórico elegido con los alcances de la metodología b-learning, al servicio de una labor de mediaciones en el aula que puede estimular el docente, en busca de la formación de lectores competentes de textos académicos. Dos de estas estrategias, justamente de las que se ocupa este texto, corresponden a la reflexión en torno al estudio de la memoria como precursora de todo proceso de comprensión de lectura y a los lecto-mitos como distractores de la acción didáctica.

\section{Metodología}

La investigación de la cual se deriva esta propuesta se desarrolla desde un enfoque eminentemente cualitativo, orientado hacia el proceso, y en respuesta a la necesidad de apoyar a los profesores en su afán de configurar estrategias eficaces para sus entornos específicos de aula. En este sentido, el grupo investigador opera en calidad de "organizador de las discusiones, facilitador del proceso, como un catalizador de problemas y conflictos y, en general, como un técnico y recurso disponible para ser consultado" (Martínez-Miguélez, 2010, 240). El desarrollo del Proyecto se llevó a cabo en tres fases, de acuerdo con la siguiente organización:

1. Planificación: descripción y preparación con las siguientes actividades:

- Fundamentación teórica. Construcción del marco teórico.

- Formación técnica del Grupo investigador acerca de la Web 2.0. Para ello, uno de los miembros del equipo investigador cursó el diplomado en Estrategias para el desempeño en ambientes virtuales de aprendizaje (192 horas).

- Diseño de cuatro módulos virtuales para el plan de intervención, a partir del marco teórico. Como fruto del proceso se construyeron las guías respectivas, de acuerdo con las directrices y los formatos establecidos por la Unidad de Virtualización de la Universidad del Quindío.

- Montaje de los módulos en la Web 2.0. Estos fueron alojados en la plataforma Moodle de la Universidad del Quindío?.

Estas estrategias son: 1. El estudio de la memoria como precursora de todo proceso de comprensión de lectura; 2. La identificación de las macrorreglas como mecanismos de recuperación textual; 3. La detección de lectomitos como distractores de la acción didáctica; 4. La familiarización con el metalenguaje de la comprensión lectora como provocación a una didáctica del funcionamiento textual; 5. La incorporación de las superestructuras como patrones de los organizadores gráficos; 6. La transposición didáctica de los niveles de representación como dispositivos para la mediación de lectura; 7. La elaboración de reseñas como género discursivo al servicio de la lectura académica; 8. El fomento de la discusión en foros virtuales como zona de desarrollo próximo para la comprensión de lectura; 9. El fortalecimiento de una conciencia hipermedial como provocadora de construcción de sentido; y 10. El despertar de una conciencia de coherencia como reguladora del ambiente virtual de aprendizaje.

9 La ruta de acceso al curso dirigido a estudiantes de Licenciatura inicia en la página www.virtual2.uniquindio. edu.co. Opción: Cursos $>$ Universidad del Quindío $>$ Facultades $>$ Educación $>$ Español y Literatura $>$ CLTA (Comprensión Lectora de Textos Académicos). 


\section{Fase experimental: Intervención con un grupo de docentes en formación:}

- Desarrollo de una intervención con 20 maestros en formación (estudiantes de $9^{\circ}$ semestre de la Licenciatura en Español y Literatura, matriculados en el espacio académico Didáctica de la comprensión y producción de textos funcionales). Esta elección permitía la aplicación en todo rigor de la metodología b-learning, pues dos profesores vinculados a la investigación orientan este curso. Aunque la duración prevista era de 4 semanas, fue preciso prolongarla a 8 por la necesidad de profundización de los temas.

- Evaluación de la calidad del proceso con el grupo de docentes en formación, base para la realización de dos trabajos de grado por parte de las dos estudiantes que intervinieron en el proyecto; tales trabajos obtuvieron calificación meritoria $^{10}$.

- Revisión y ajustes a los módulos virtuales, a partir de la experiencia con el grupo de docentes en formación.

- Edición de la plataforma interactiva con los productos diseñados. Para ello, el equipo de la Unidad de Virtualización de la Universidad del Quindío abrió en plataforma una nueva versión del curso de Comprensión Lectora de Textos Académicos (CLTA_2015-1) ${ }^{11}$.

- Implementación de la intervención con 15 maestros en ejercicio de Educación Básica (estudiantes de la Maestría en Ciencias de la Educación, que participaban en los seminarios Comprensión y producción de textos científicos, y Seminario de trabajo de grado I).

\section{Redacción y difusión del proyecto:}

- Análisis de resultados.

- Evaluación del proyecto.

- Conclusiones.

- Elaboración de informe final.

- Socialización en eventos académicos de los resultados del proyecto.

10 Se trata de los trabajos Estrategias de integración del marco situacional, al servicio de la comprensión lectora de textos académicos mediada por la plataforma Moodle, en un grupo de estudiantes de licenciatura en Español y Literatura de la Universidad del Quindio (2014-2015) de Olga Johanna Jaramillo y Análisis de mapas conceptuales y resúmenes para comprensión de textos expositivo-explicativos a través de la plataforma Moodle, en docentes en formación de la Universidad del Quindio (2014-2015) de Johanna Andrea Ordóñez Ruiz.

11 La ruta de acceso al curso dirigido a estudiantes de la Maestría parte de la página www.virtual2.uniquindio.edu. co. Opción: Cursos $>$ Universidad del Quindío $>$ Facultades $>$ Educación $>$ Maestría en Ciencias de la Educación $>$ CLTA_2015-1 (Comprensión Lectora de Textos Académicos 2015-1). 
En cuanto a la estructura de los módulos virtuales, el desarrollo fue el siguiente:

\begin{tabular}{|c|c|c|c|c|}
\hline \multicolumn{5}{|c|}{ Intervención dirigida a docentes en formación } \\
\hline & Nombre & $\begin{array}{l}\text { Pregunta problemati- } \\
\text { zadora }\end{array}$ & Contenidos & $\begin{array}{l}\text { Interactividades e interac- } \\
\text { ciones }^{12}\end{array}$ \\
\hline 0 & $\begin{array}{l}\text { Ambientación } \\
\text { y diagnóstico }\end{array}$ & $\begin{array}{l}\text { ¿Qué fortalezas y de- } \\
\text { bilidades se advierten } \\
\text { en la comprensión de } \\
\text { textos académicos? }\end{array}$ & 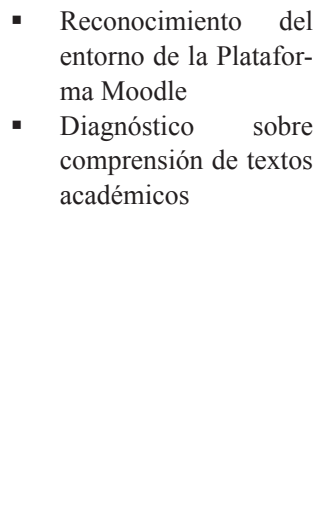 & 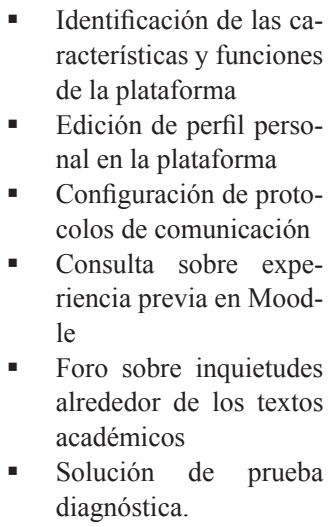 \\
\hline 1 & $\begin{array}{l}\text { El concepto de } \\
\text { texto y sus ca- } \\
\text { racterísticas }\end{array}$ & $\begin{array}{l}\text { ¿Cuáles son los mitos } \\
\text { que se han creado en } \\
\text { torno al concepto de } \\
\text { texto y cómo pueden } \\
\text { refutarse a la luz de lo } \\
\text { estudiado? }\end{array}$ & $\begin{array}{l}\text { - Mitos y verdades en } \\
\text { torno al concepto de } \\
\text { texto. } \\
\text { - Las características del } \\
\text { texto académico en } \\
\text { cuanto a sus dimen- } \\
\text { siones: } \\
\text { - Notacional } \\
\text { - Morfológica } \\
\text { - Sintáctica } \\
\text { - Semántica } \\
\text { - Pragmática } \\
\text { Las características } \\
\text { del texto académico } \\
\text { en cuanto a sus ni- } \\
\text { veles: } \\
\text { - Superestructural } \\
\text { - Macroestructural } \\
\text { - Microestructural } \\
\text { - Estilístico } \\
\text { - } \quad \text { Retórico }\end{array}$ & $\begin{array}{l}\text { Foro: Hacia una defini- } \\
\text { ción de texto } \\
\text { Foro: Dudas e inquietu- } \\
\text { des sobre dimensiones y } \\
\text { niveles del texto } \\
\text { Tarea: Análisis del com- } \\
\text { portamiento de un texto } \\
\text { académico en cuanto a } \\
\text { dimensiones y niveles } \\
\text { Crucigrama interactivo: } \\
\text { Zona de desafíos tex- } \\
\text { tuales } \\
\text { Foro sobre inquietudes } \\
\text { alrededor de los textos } \\
\text { académicos }\end{array}$ \\
\hline
\end{tabular}

12 De acuerdo con los lineamientos de la Universidad del Quindío condensados en el libro Estrategia Virtual. Una alternativa para los procesos de enseñanza aprendizaje (2011), las interactividades tienen que ver con la mediación de contenidos, mientras que las interacciones se refieren a la comunicación entre estudiante-profesor, estudiante-estudiante y estudiante-institución (Universidad del Quindío, 2011, 11). 


\begin{tabular}{|c|c|c|c|c|}
\hline 2 & $\begin{array}{l}\text { El proceso de } \\
\text { comprensión de } \\
\text { lectura }\end{array}$ & $\begin{array}{l}\text { ¿Cómo se puede ca- } \\
\text { racterizar el proceso de } \\
\text { comprensión lectora, } \\
\text { a la luz de los mode- } \\
\text { los interactivos y del } \\
\text { enfoque sociocultural } \\
\text { cognitivo? }\end{array}$ & $\begin{array}{l}\text { Características del mo- } \\
\text { delo constructivo-inte- } \\
\text { grativo de Kintsch y } \\
\text { van Dijk (1978). } \\
\text { - Niveles de represen- } \\
\text { tación en el procesa- } \\
\text { miento de la informa- } \\
\text { ción textual, según } \\
\text { Kintsch (1998): } \\
\text { - Código de super- } \\
\text { ficie } \\
\text { - Texto base } \\
\text { - Modelo de situa- } \\
\text { ción }\end{array}$ & $\begin{array}{l}\text { Foro: Construcción de } \\
\text { esquemas sobre el mo- } \\
\text { delo constructivo-inte- } \\
\text { grativo } \\
\text { Cuestionario interac- } \\
\text { tivo: Ideas principales } \\
\text { sobre el modelo cons- } \\
\text { tructivo-integrativo } \\
\text { Construcción de una } \\
\text { Wiki: Taller de com- } \\
\text { prensión lectora en } \\
\text { torno a un texto, de } \\
\text { acuerdo con el modelo } \\
\text { constructivo-integrativo }\end{array}$ \\
\hline 3 & $\begin{array}{l}\text { La tipología } \\
\text { textual expositi- } \\
\text { vo-explicativa }\end{array}$ & $\begin{array}{l}\text { ¿Cuáles son las carac- } \\
\text { terísticas de la tipo- } \\
\text { logía textual exposi- } \\
\text { tivo-explicativa y las } \\
\text { estrategias cognitivas } \\
\text { y metacognitivas que } \\
\text { pueden cualificar su } \\
\text { comprensión? }\end{array}$ & $\begin{array}{l}\text { Generalidades del tex- } \\
\text { to expositivo-explica- } \\
\text { tivo. } \\
\text { - Subtipos del texto ex- } \\
\text { positivo-explicativo } \\
\text { Características y mar- } \\
\text { cas lingüísticas de los } \\
\text { textos expositivo-ex- } \\
\text { plicativos }\end{array}$ & $\begin{array}{l}\text { - Foro: Generalidades del } \\
\text { texto expositivo-expli- } \\
\text { cativo } \\
\text { Foro: Exposición de } \\
\text { ejemplos para cada sub- } \\
\text { tipo de texto expositi- } \\
\text { vo-explicativo } \\
\text { - Construcción de glosario } \\
\text { interactivo: Conceptos } \\
\text { clave en torno al texto } \\
\text { expositivo-explicativo. }\end{array}$ \\
\hline \multicolumn{5}{|c|}{ Intervención dirigida a docentes en ejercicio } \\
\hline & Nombre & $\begin{array}{l}\text { Pregunta problemati- } \\
\text { zadora }\end{array}$ & Contenidos & $\begin{array}{l}\text { Interactividades e interac- } \\
\text { ciones }\end{array}$ \\
\hline 0 & $\begin{array}{l}\text { Ambientación y } \\
\text { diagnóstico }\end{array}$ & $\begin{array}{l}\text { ¿Qué fortalezas y de- } \\
\text { bilidades se advierten } \\
\text { en la comprensión de } \\
\text { textos académicos? }\end{array}$ & $\begin{array}{l}\text { Reconocimiento del } \\
\text { entorno de la Platafor- } \\
\text { ma Moodle } \\
\text { - Inquietudes alrededor } \\
\text { de los textos académi- } \\
\cos ^{13}\end{array}$ & $\begin{array}{l}\text { - Identificación de las ca- } \\
\text { racterísticas y funciones } \\
\text { de la plataforma } \\
\text { - Edición de perfil perso- } \\
\text { nal en la plataforma } \\
\text { - Configuración de proto- } \\
\text { colos de comunicación } \\
\text { - Foro sobre inquietudes } \\
\text { alrededor de los textos } \\
\text { académicos. }\end{array}$ \\
\hline 1 & $\begin{array}{l}\text { La tipología } \\
\text { textual expositi- } \\
\text { vo-explicativa }\end{array}$ & $\begin{array}{l}\text { ¿Cuáles son las carac- } \\
\text { terísticas de la tipo- } \\
\text { logía textual exposi- } \\
\text { tivo-explicativa y las } \\
\text { estrategias cognitivas } \\
\text { y metacognitivas que } \\
\text { pueden cualificar su } \\
\text { comprensión? }\end{array}$ & $\begin{array}{l}\text { Generalidades del tex- } \\
\text { to expositivo-explica- } \\
\text { tivo. } \\
\text { - Subtipos del texto ex- } \\
\text { positivo-explicativo } \\
\text { - Características y mar- } \\
\text { cas lingüísticas de los } \\
\text { textos expositivo-ex- } \\
\text { plicativos }\end{array}$ & $\begin{array}{l}\text { - Foro: Generalidades del } \\
\text { texto expositivo-explicativo } \\
\text { - Foro: Exposición de } \\
\text { ejemplos para cada sub- } \\
\text { tipo de texto expositi- } \\
\text { vo-explicativo } \\
\text { - Construcción de glosario } \\
\text { interactivo: Conceptos } \\
\text { clave en torno al texto } \\
\text { expositivo-explicativo }\end{array}$ \\
\hline
\end{tabular}

13 De la intervención dirigida a docentes en formación se omitió el diagnóstico sobre comprensión de textos académicos, ya que para ese momento, en el espacio académico de Comprensión y producción de textos cientificos, 


\section{Resultados y discusión}

Tal como lo indicamos en la introducción, en este artículo nos ocupamos de dos de las diez estrategias que surgieron del proyecto base. A continuación, las explicamos en detalle:

\subsection{El estudio de la memoria como precursora de todo proceso de comprensión de lectura}

Si se concibe la didáctica, desde el pensamiento de Chevallard (1991), como disciplina de intervención que responde a enfoques teóricos definidos en torno al docente, el estudiante y el saber, el respaldo de todo abordaje sobre los procesos de comprensión de lectura debe partir del estudio de la memoria, en cuanto a sus características, tipos, limitaciones, duración y capacidad de almacenamiento. Sería imposible imaginar estrategias para la formación de lectores competentes de espaldas a las condiciones propias del cerebro humano. Un ejemplo del reconocimiento de tal necesidad viene de Teun van Dijk, cuando en su autobiografía académica (2006) justifica su acercamiento a Walter Kintsch, en nombre de una psicología del procesamiento del texto:

Los usuarios de la lengua representan oraciones y sus significados en su memoria. Es decir, una teoría psicológica es una teoría del proceso mental y necesita tener en cuenta que, por ejemplo, nuestro "memoria de trabajo", es decir, la Memoria a Corto Plazo (MCP) tiene una capacidad limitada y necesita vaciarse regularmente después de lo cual la información interpretada se almacena en la Memoria a Largo Plazo (MLP) (van Dijk, 2006, 8).

Así, el ejemplo del lingüista que busca apoyo del psicólogo para entender el fenómeno de la lectura es modelo emblemático para el maestro de lengua materna, que no podría formar lectores competentes sin incursionar antes en lo que dictan los estudios de la neurociencia sobre el modo como funciona el cerebro, ya que, en última instancia, el acto de leer no se cristaliza si no trasciende las fronteras de los siete u ocho bits de la MCP hasta llegar a la MLP. De este modo, algunas preguntas problematizadoras que agenciarían esta estrategia serían: ¿Cómo supera el lector las limitaciones de la MCP al enfrentarse con información nueva en el texto? ¿Cómo ayuda el texto a que el lector alcance a llevar la información nueva a la MLP ${ }^{14}$ ? ¿Qué debe hacer el lector ante textos que rebasen las posibilidades de su MCP? ¿Cómo se manifiestan los recursos de la repetición, el agrupamiento de bits y la asociación en la cohesión textual, en su calidad de mecanismos que facilitan el paso de la MCP a la MLP? ¿Cómo regula el texto las relaciones tema/rema ${ }^{15}$ ? ¿Qué debe hacer el

ya se había realizado dicho trabajo, de modo enteramente presencial. De ahí que solo se retomará el foro sobre inquietudes. Por la misma razón, se omitió la unidad dedicada al concepto de texto y sus características. Se aclara, entonces, que no se trata propiamente de una omisión, sino de un avance en el seminario presencial anterior.

14 Esto dialogaría directamente con el principio de cooperación de Grice: "Haga que su contribución a la conversación sea, en cada momento, la requerida por el propósito o la dirección del intercambio comunicativo en el que está usted involucrado" (1983, 105).

15 La informatividad corresponde a la quinta norma de textualidad que proponen De Beaugrande y Dressler (1997). 
lector para evitar una saturación de la MCP mientras accede a información nueva? ¿Cómo se articulan la memoria semántica y episódica en la construcción de sentido que hace el lector del texto? Estas y otras preguntas del mismo tenor rompen con la visión accesoria o instrumental de las estrategias de lectura -concebidas como recetas automatizadas-y las elevan de rango, esta vez, al servicio de una conciencia metacognitiva que monitorea los avances de la comprensión, desde las condiciones particulares y complejas del cerebro que lee.

En el proyecto de investigación, esta estrategia tuvo lugar esencialmente en las interacciones presenciales, como parte de la fundamentación teórica previa, tanto con los docentes en formación como con los docentes en ejercicio ${ }^{16}$. Se sintetizan en el siguiente cuadro algunos hechos que se priorizaron en el marco de esta estrategia. Se trata de relaciones puntuales entre prácticas estratégicas concretas y los fenómenos de orden cognitivo que les dan fundamento. Tales relaciones salieron a flote en las interacciones presenciales y se reforzaron en los foros virtuales de discusión, así:

\begin{tabular}{|c|c|}
\hline Prácticas estratégicas & Fenómeno cognitivo asociado \\
\hline 1. Macrorregla de la supresión & $\begin{array}{l}\text { Limitación de la capacidad de almacenamiento de la } \\
\text { MCP ( } 7 \text { u } 8 \text { bits })\end{array}$ \\
\hline 2. Macrorregla de la generalización & $\begin{array}{l}\text { Agrupamiento de bits para rentabilizar el almacena- } \\
\text { miento en la MCP }\end{array}$ \\
\hline 3. Cohesión por hiperonimia & $\begin{array}{l}\text { Agrupamiento de bits para rentabilizar el almacena- } \\
\text { miento en la MCP }\end{array}$ \\
\hline 4. Macrorregla de la construcción & $\begin{array}{l}\text { Mecanismo de la asociación para rentabilizar el } \\
\text { paso de información de la MCP a la MLP }\end{array}$ \\
\hline 5. Elaboración de inferencias & $\begin{array}{l}\text { Mecanismo de la asociación para rentabilizar el } \\
\text { paso de información de la MCP a la MLP }\end{array}$ \\
\hline 6. Cohesión por repetición & $\begin{array}{l}\text { Mecanismo de la repetición para rentabilizar el paso } \\
\text { de información de la MCP a la MLP }\end{array}$ \\
\hline $\begin{array}{l}\text { 7. Inclusión de elementos paraverbales } \\
\text { (color, tamaño, formato, tipo de le- } \\
\text { tra, recuadros...) en los organizado- } \\
\text { res gráficos }\end{array}$ & Estímulo de la memoria sensorial y viso-espacial \\
\hline 8. Lectura a la luz de un problema & Relaciones entre emoción, atención y memoria \\
\hline $\begin{array}{l}\text { 9. Identificación de palabras claves del } \\
\text { texto }\end{array}$ & $\begin{array}{l}\text { Estimulación de la memoria semántica y de la me- } \\
\text { moria de trabajo }\end{array}$ \\
\hline 10. Sondeo de conocimientos previos & Activación de la memoria de trabajo \\
\hline
\end{tabular}

Sirve para evaluar hasta qué punto las secuencias de un texto transmiten información conocida o novedosa. Esto significa que el concepto de informatividad se relaciona con el grado de novedad o de imprevisibilidad que tiene un texto para sus receptores y se valora en función de su contenido.

16 Para la primera, el espacio académico presencial era el de Didáctica de la comprensión y producción de textos funcionales (Licenciatura en Español y Literatura, 2014-II), mientras que para la segunda, tres espacios sirvieron a dicho propósito: Comprensión y producción de textos científicos; Didáctica de la comprensión y producción de textos y sus géneros discursivos; y Seminario de Trabajo de Grado I (Maestría en Ciencias de la Educación, 2015-I). 


\begin{tabular}{|l|l|}
\hline $\begin{array}{l}\text { 11. Reconocimiento del propósito de } \\
\text { lectura }\end{array}$ & $\begin{array}{l}\text { Delimitación de campos de atención a la hora de } \\
\text { leer y facilitar el olvido de la información no perti- } \\
\text { nente para el propósito }\end{array}$ \\
\hline 12. Diseño de una ruta de trabajo & $\begin{array}{l}\text { Aseguramiento de la estrategia de coherencia, gra- } \\
\text { cias a la identificación de nodos en la memoria de } \\
\text { trabajo y sus relaciones entre sí }\end{array}$ \\
\hline
\end{tabular}

Afirman Smith y Kosslyn que "sin el conocimiento en sus varios papeles, en la categorización y la deducción, en la acción, en la percepción y atención, en la memoria, en el lenguaje y en el pensamiento, seríamos zombis en la fiesta" $(2008,156)$. Bien se podría extender la sentencia al proceso de enseñanza de la lectura de textos académicos: si el maestro no tiene en cuenta los complejos procesos asociados a la cognición humana antes de pensar en las estrategias para la comprensión, puede estar formando -acaso sin saberlo-zombis en el aula.

\subsection{La detección de lecto-mitos como distractores de la acción didáctica}

En el afán por desentrañar el papel de la memoria en los procesos de comprensión de lectura, poco a poco, la dinámica investigativa deja al descubierto los errores que, al abrigo de la misma tradición, perviven como inmodificables en las prácticas docentes. Podríamos decir que, del mismo modo como las prácticas educativas se inundan de neuromitos ${ }^{17}$ patrocinados por lecturas imprecisas o sesgadas de los avances investigativos en los estudios del cerebro, al parecer la didáctica de la lengua también es terreno propicio para que germinen diversos lecto-mitos que hacen carrera hasta convertirse en pieza clave de los imaginarios pedagógicos docentes, alrededor de la enseñanza de la lectura. En este proyecto de investigación se pudieron identificar cuatro: el de la lectura como simple decodificación, el de la lectura como simple subrayado, el de la lectura como simple extracción de ideas y el de la lectura como simple práctica monofónica (o "leer a secas") ${ }^{18}$.

a. La lectura como simple decodificación. Heredera de los modelos ascendentes y auspiciada por un paradigma tradicional que considera al lenguaje como simple suma de elementos (letras, sílabas, frases, oraciones, párrafos...), la concepción predominante en los diagnósticos sobre lectura corresponde a leer como sinónimo de decodificar, una tarea mecánica que -tal como lo refiere la acepción de la $23^{\mathrm{a}}$ versión del Diccionario de la Real Academia (2014)- consiste en "aplicar inversamente las reglas de su código a un mensaje codificado para obtener la forma primitiva de este". Asumir tal sinonimia entre lectura y decodificación excluiría por completo al sujeto desde sus conocimientos previos, su memoria episódica y semántica, sus afectos y su protagonismo en la construcción de sentido, lo que reviviría la ya superada visión

17 Los neuromitos son definidos por la OCDE como "las trampas que surgen cuando se establecen puentes equivocados o sin fundamento entre la neurociencia y la educación” (2009, 169); por ejemplo, el neuromito de Gall, según el cual era posible distinguir entre un criminal y un hombre honrado por la forma de su cerebro.

18 Esta propuesta dialoga de modo muy cercano con las concepciones y prácticas sobre la lectura que describe Sánchez (2014, 9-22). El autor agrupa estas concepciones a modo de dilemas, así: 1. Leer es decodificar vs. Leer es construir sentido; 2. Lectura como producto vs. Lectura como proceso; 3. Todos los textos se leen igual vs. Lectura eferente y lectura estética; 4. Se lee en clase de lenguaje vs. Se lee en todas las áreas. 
reproductiva del texto al servicio de un lenguaje monológico. Este lecto-mito arrastra consigo diversas aberraciones didácticas; Sánchez $(2014,12)$ describe una de ellas: "Lo problemático en algunas aulas es que los estudiantes leen todos los textos de la misma manera, pues los propósitos no suelen ser claros, ni significativos, ni diversos. Lo que predomina es leer para responder preguntas que supuestamente determinan si un estudiante entendió o no".

b) La lectura como simple subrayado. A la falsa sinonimia de leer/codificar, se añade la de leer/subrayar. Este lecto-mito emergió en medio de las interacciones presenciales y virtuales alrededor de la práctica del resumen. Tres tendencias lo revelaron: 1. Entregar como resumen el texto subrayado; 2. Presentar el resumen como lista de ideas que se trascriben literalmente del texto; 3. Mantener las voces enunciativas del texto original en el resumen. En suma, para la mayoría de los docentes que participaron en el proyecto, el resumen acababa en el subrayado, lo que excluía por completo el marco situacional, pieza vital en el modelo constructivo-integrativo. Esta creencia, que se alimenta de la visión fragmentaria heredada de modelos ascendentes, bloquea de modo definitivo el acercamiento de los docentes a modelos descendentes $\mathrm{y}$, ante todo, a los interactivos (entre los cuales se cuenta el constructivo-integrativo de Kintsch y van Dijk). Más aún, deja sin piso cualquier reflexión no instrumental en favor de la lectura; por ejemplo, aquella que recupera Fernando Vásquez $(2000,88)$ de los trabajos interpretativos de Estanislao Zuleta sobre la obra de Nietzsche: "En un principio hay que "cargar" con el texto, volverlo familia, rumiarlo, digerirlo, permitirle hibernar en nuestra conciencia; luego hay que enfrentarse a él, desconfiar, ponerlo entre paréntesis, discutirlo; finalmente debemos tratar de producir un texto nuevo, diferente, generar otros textos".

c) La lectura como simple extracción de ideas. Al leer/codificar y al leer/subrayar, se suma el leer/extraer. Este nuevo lecto-mito se advierte tras los intersticios de una consigna muy común entre los docentes participantes en los foros: extraer las ideas principales de un texto. Es tan recurrente su presencia en talleres y evaluaciones, que podría decirse que ya forma parte de los imaginarios de enseñanza de la lectura. Tal como ocurre con el lecto-mito anterior, la práctica lectora del extraer alcanza a llegar, a lo sumo, hasta las instancias del texto-base y olvida por completo la necesidad de un marco situacional que se integra desde los conocimientos previos del lector. Si en una didáctica de los procesos de comprensión, el estudiante solo extrae ideas, los textos se convierten en un artículo más de consumo y pierden su función dialógica, su comunicación en doble vía, la construcción que propician de la imagen de $s u$ lector; en última instancia, si en estas dinámicas monológicas de aula, leer es extraer, enseñar será solo transmitir.

d) La lectura como simple práctica monofónica: Este lecto-mito es de largo aliento en la historia de las prácticas escolares y solo en las últimas dos décadas viene perdiendo su fuerza, gracias al permanente debate de los círculos académicos (adscritos a movimientos como leer y escribir a través del currículo o de alfabetización académica), en torno a las tipologías textuales y géneros discursivos ${ }^{19}$. Deviene, quizás, de una interpretación sesgada de la propuesta de construcción de las cuatro habilidades básicas (leer, escribir, hablar y escuchar). En el afán por favorecer la enseñanza de lo

19 En este sentido, la investigación se adscribe a la perspectiva del interaccionismo socio-discursivo: "Los géneros son formas comunicativas (novela, editorial, enciclopedia, etcétera) que se pondrán en correlación con las unidades psicológicas que constituyen las acciones verbales, mientras que los tipos de discurso (narración, discurso 
comunicativo, la escuela benefició el mito de la lectura como práctica universalizada (en singular y precedida de artículo definido). Podría decirse que leer se convirtió en una abstracción regida por leyes (y estrategias) nomotéticas ${ }^{20}$, bien lejana de la diversidad textual (idiográfica por naturaleza) que define superestructuras, estilísticas, gramáticas y retóricas particulares y, en última instancia, estrategias específicas para su comprensión y producción; por ejemplo, no es lo mismo elaborar un resumen sobre un texto expositivo-explicativo que hacerlo sobre uno argumentativo, ya que la aplicación de macrorreglas como la de supresión-según van Dijk $(1983,49)$-depende del tipo de texto ${ }^{21}$. En otras palabras, el lecto-mito de leer como práctica monofónica se derrumba con la polifonía de los géneros; por eso, en lugar del verbo - universal, monocorde, monofónico y generalísimo-leer, la didáctica de la lengua prefiere los específicos -situados, policromados, polifónicos y específicos-narrar, describir, argumentar, exponer, explicar, debatir, reseñar, recitar, discutir, informar, disertar...

Una muestra de la manera de enfrentar estos lecto-mitos se ilustra a continuación, desde un derrotero para explicar, modelar y acompañar la elaboración de un resumen, en sintonía con las propuestas de Álvarez (2013) 22 :

\begin{tabular}{|c|c|c|}
\hline & Para texto expositivo-explicativo & Para texto argumentativo \\
\hline $\begin{array}{l}2 . \\
3 .\end{array}$ & $\begin{array}{l}\text { Identificación del subtipo (definición-descripción, } \\
\text { clasificación-tipología, comparación-contraste, } \\
\text { causa-consecuencia, problema-solución, pregun- } \\
\text { ta-respuesta) } \\
\text { Descripción del propósito del texto } \\
\text { Análisis y selección de la información: dividir el } \\
\text { texto en párrafos y localizar la idea central de cada } \\
\text { uno, mediante la aplicación de macrorreglas } \\
\text { Eliminación de la información redundante o no } \\
\text { sustancial, mediante una nueva aplicación de ma- } \\
\text { crorreglas } \\
\text { Jerarquización de las ideas } \\
\text { Definición de las inferencias o sobreentendidos } \\
\text { (para esto, sirve mucho la macrorregla de construc- } \\
\text { ción) } \\
\text { Reescritura del texto uniendo cohesivamente las } \\
\text { ideas conseguidas }\end{array}$ & $\begin{array}{l}\text { 1. Identificación de la tesis del autor (su propósito } \\
\text { central) } \\
\text { 2. Análisis y selección de la información: dividir el } \\
\text { texto en párrafos y localizar la idea central de cada } \\
\text { uno, mediante la aplicación de macrorreglas. En } \\
\text { este caso, se trata de las conclusiones (afirmacio- } \\
\text { nes u opiniones a favor de las cuales está el autor) } \\
\text { 3. Eliminación de la información redundante o no } \\
\text { sustancial mediante una nueva aplicación de ma- } \\
\text { crorreglas } \\
\text { 4. Jerarquización de las ideas } \\
\text { 5. Definición de las inferencias o sobreentendidos } \\
\text { (para esto, sirve mucho la macrorregla de cons- } \\
\text { trucción). } \\
\text { 6. Reescritura del texto uniendo cohesivamente las } \\
\text { ideas conseguidas }\end{array}$ \\
\hline
\end{tabular}

teórico, etcétera) serán considerados como formas lingüísticas más específicas que entran en la composición de los géneros" (Bronckart, 2004, 15). Recuerda Bronckart (2007) que los géneros, como hechos sociales, cambian con el tiempo o con la historia de las formaciones socioverbales, sometidos a indexaciones referenciales, comunicativas y culturales; asimismo, expresa la imposibilidad de una clasificación estable y definitiva de los géneros.

20 Se presta aquí el término nomotético (en oposición a lo ideográfico) de la discusión en Ciencias Sociales; lo nomotético -según Santos $(2006,68)$ - hace alusión a disciplinas como la sociología, economía y ciencias políticas que aspiraban a encontrar leyes universales para explicar lo social, mediante el método predicción/control/ explicación.

21 Para dar más detalles al respecto, la inclusión o no de una fecha en un resumen depende del tipo de texto. En uno expositivo-explicativo que intente recuperar la cronología y evolución de un concepto, será vital; mientras que en uno argumentativo, en el que este dato solo sirva para reforzar la veracidad del argumento, sería prescindible.

22 Propone el autor 5 fases para la práctica del resumen: 1. Comprensión del texto; 2. Localización de las ideas importantes o principales; 3. Ordenación de las ideas; 4. Supresión de todas las palabras posibles; y 5. Reescritura del texto $(2013,59)$. 
Con este tipo de propuestas, se podrían desestabilizar conceptual y metodológicamente los lecto-mitos antes descritos. El primer lectomito (La lectura como simple decodificación) queda sin sustento con la insistencia, desde el inicio, en la revisión de propósitos y de elementos superestructurales. El segundo (La lectura como simple subrayado) y el tercero (La lectura como simple extracción de ideas) se derrumban con los pasos que siguen a la identificación de ideas principales: nueva poda de ideas principales, jerarquización, desarrollo de inferencias y re-escritura. Y el último (La lectura como simple práctica monofónica) se erradica desde rutas distintas de trabajo, según la tipología textual, lo que podría afinarse con detalles más precisos relacionados con el género discursivo en particular. De este modo, se aspira a estimular en los docentes una actitud de vigilancia epistemológica atenta al crecimiento de mitos - esta vez en la lectura, pero detectables en todo proceso- que, al estilo de bolas de nieve, cobran fuerza en las prácticas educativas y obstaculizan las acciones renovadoras.

\section{Conclusiones}

El proyecto de investigación Estrategias para lograr lectores competentes de textos académicos. Un programa interactivo de intervención didáctica dirigido a profesores de Educación Básica en el Departamento del Quindío cumplió a cabalidad con los objetivos fijados. En efecto, diseñó un programa interactivo en el ambiente de la plataforma Moodle de la Universidad del Quindío y lo aplicó con dos grupos: uno de docentes en formación (adscritos a la Licenciatura en Español y Literatura) y otro de docentes en ejercicio (pertenecientes a la Maestría en Educación), a través de la metodología B-learning. Evaluada la calidad de las interactividades e interacciones propiciadas, y analizados los resultados que se desprenden del apartado anterior, se destaca el balance favorable en los frentes conceptual, procedimental y actitudinal, lo que hace factible pensar en la posibilidad de formar primero a los docentes, con la esperanza de que en su práctica profesional impacten de modo positivo los procesos de comprensión lectora de sus estudiantes.

De todos modos, no podría pensarse que el proyecto aquí se termine. Precisamente, la elección de estas dos poblaciones obedece a la posibilidad de acompañarlas, por lo menos, durante dos años más, en ámbitos definitivos como la práctica docente, la investigación en el aula y la definición y desarrollo de sus trabajos de grado, ya que los miembros del equipo investigador concentran sus labores de docencia en estos dos programas académicos. Solo desde esos resultados a largo plazo podría, en verdad, dictaminarse el éxito de esta iniciativa. Paralelamente, desde el impulso de esta experiencia positiva, los investigadores pretenden no solo socializar los hallazgos del proyecto, sino replicarlo en otros círculos de docentes en ejercicio, esta vez más alejados del contexto de formación universitaria, con el fin de fortalecer cada frente estratégico aquí propuesto y de articularlo con otros que se han derivado de anteriores investigaciones ${ }^{23}$.

23 Por ejemplo, del proyecto Análisis de la producción escrita de estudiantes de grados 5 y 9 de Educación Básica en el marco de la preparación para las pruebas Saber en la ciudad de Armenia y en el Departamento del Quindio (Grupo DiLeMa, 2011-2012) emergieron las siguientes estrategias: 1. La escritura desde el paso por las dimensiones y niveles del texto; 2. Escritura, revisión de borradores y textos intermedios; 3 . Hacia una didác- 
A la luz de las anteriores consideraciones, avanzar en la superación de lecto-mitos de aula supone cambios profundos en las maneras como los docentes de hoy asumen sus compromisos de mediadores de lectura de los textos de su disciplina; en ese intento sería propicio estimular el despertar de lo que aquí se ha defendido como conciencia constructivo-integrativa que iría más allá de los ámbitos conceptual y procedimental, hasta instalarse en el actitudinal.

De hecho, iniciar por una transposición didáctica de los niveles de representación, visible en los talleres, conversatorios, ejercicios y tareas que el docente proponga desde el aula, sería un paso importante en procura de dicho estado de conciencia, gracias al cual sea posible superar estos y otros lecto-mitos que cobran vida en la escuela. Desde propuestas de intervención que los enfrenten, podría hacerse realidad aquella sentencia de Bronckart $(2007,98)$, imprescindible para la consolidación de una didáctica de la lengua: "toda metodología es también una opción epistemológica", lo que en contextos de aula se traduciría en que detrás de toda actuación docente debe advertirse un respaldo teórico; al fin y al cabo, la didáctica, en su calidad de utopía indispensable desde el pensamiento de Bronckart y Schneuwly (1996), sería, ante todo, teoría en acción.

\section{Bibliografía}

Adam, J. M. (1992) : Les textes: types et prototypes. Récit, description, argumentation, explication, dialogue, Paris, Nathan.

Álvarez, T. (2001): Textos expositivo-explicativos y argumentativos, Barcelona, Octaedro.

Álvarez, T. (2013): Didáctica de la lengua para la formación de maestros, Barcelona, Octaedro.

Bachman, L. (1995): "Habilidad lingüística comunicativa", en Llobera, M. (coord.), Competencia comunicativa: documentos básicos en la enseñanza de lenguas extranjeras, Madrid, Edelsa, 105-128.

Beaugrande, R. y W. Dressler (1997): Introducción a la lingüistica del texto, Barcelona, Ariel.

Bronckart, J. P. (1996): Activité langagière, textes et discours. Pour un interactionisme socio-discursif, Lausanne, Delachaux et Niestlé.

Bronckart, J. P. (2004): Actividad verbal, textos y discursos: Por un interaccionismo socio-discursivo, Madrid, Fundación Infancia y Aprendizaje.

tica de la escritura desde la línea psicológica de la argumentación; 4. Hacia una didáctica de la escritura desde la línea lógica de la argumentación; 5. Escritura y conciencia del topos; 6. La escritura desde una perspectiva apolínea; 7. La escritura desde una perspectiva dionisíaca; 8. La escritura en los movimientos tema-rema; 9. La escritura y la recuperación del concepto de párrafo; 10. La escritura y la conciencia de los modalizadores. Asimismo del proyecto Estrategias de comprensión y producción de textos argumentativos desde un enfoque sociocultural. Una propuesta didáctica dirigida a profesores de Educación Básica para el mejoramiento de la competencia lectoescritural de sus estudiantes (Grupo DiLeMa, 2008-2009) se derivaron las siguientes estrategias: 1. Promoción de la lectura comprensiva de textos antitéticos; 2. Estímulo a la escritura de textos antitéti$\cos ; 3$. Fomento del análisis de la retórica de discursos mediáticos; 4. Abordaje de textos argumentativos tácitos (con sentidos escondidos); 5. Rescate del análisis del hecho retórico, como precursor de la escritura de textos argumentativos; 6. Recuperación de la dispositio en la construcción de textos argumentativos; 7. Recuperación del género epistolar con intenciones argumentativas; 8. Redención del avatar del lector-camello; 9. Resignificación del género discursivo del debate en el aula de clase; 10. Persistencia en la evaluación por criterios cualitativos. 
Bronckart, J. P. (2007): Desarrollo del lenguaje y didáctica de las lenguas, Buenos Aires, Miño y Dávila.

Bronckart, J. P. y B. Schneuwly (1996): "La didáctica de la lengua materna: el nacimiento de una utopía indispensable", Textos de didáctica de la lengua y la literatura, 9, 61-78.

Camps, A. (2004): “Objeto, modalidades y ámbitos de la investigación en didáctica de la lengua", Lenguaje, 32, 7-27.

Canale, M. y M. Swain (1980): "Fundamentos teóricos de los enfoques comunicativos. La enseñanza y la evaluación de una segunda lengua", Signos, teoría y práctica de la educación, 17, 54-62.

Chevallard, Y. (1991): La transposición didáctica, Argentina, Aique.

Dijk, T. A. van (1978): La ciencia del texto, Barcelona, Paidós.

Dijk, T. A. van (1983): Estructuras y funciones del discurso, México, Siglo XXI.

Dijk, T. A. van (2006): De la gramática del texto al análisis crítico del discurso. Una breve autobiografía académica. http://www.discursos.org/cv/ [Consulta: 11 julio 2015].

Dijk, T. A. van, y W. Kintsch (1978): "Towards a model of discourse comprehension and production", Psychological Review, 85, 363-394.

Dijk, T. A. van, y W. Kintsch (1983): Strategies of Discourse Comprehension, Nueva York, Academic Press.

Gaskins, I. y T. Elliot (1999): Cómo enseñar estrategias cognitivas en la escuela. El manual Benchmark para docentes, Buenos Aires, Paidós.

Grice, P. (1983): “Lógica y Conversación”, en Lenguaje y Sociedad, Cali, Centro de Traducciones, Universidad del Valle, 101-121.

Halliday, M. A. K. (1982): El lenguaje como semiótica social. La interpretación social del lenguaje y del significado, México, Fondo de Cultura Económica.

Hymes, D. (1967): "Studying the Interaction of Language and Social Life", en Foundation in Sociolinguistics. An Ethnographic Approach, London, Tavistock, 29-66.

Hymes, D. (1995): “Acerca de la competencia comunicativa”, en Llobera, M. (coord.), Competencia Comunicativa documentos básicos en la enseñanza de lenguas extranjeras, Madrid, Edelsa, 27-46.

Kintsch, W. (1998): Comprehension. A paradigm for cognition, Cambridge, University Press.

Kintsch, W. y J. Yarborough (1982): "The Role of Rhetorical Structure in Text Comprehension", Journal of Educational Psychology, 74, 828-834.

Martínez-Miguélez, M. (2010): Ciencia y arte en la metodología cualitativa, México, Trillas.

Monereo, C. y J. I. Pozo (eds.) (2003): La universidad ante la nueva cultura educativa. Enseñar y aprender para la autonomía, Madrid, Síntesis.

OCDE (2009): La comprensión del cerebro. El nacimiento de una ciencia del aprendizaje, Santiago, Universidad Católica Silva Henríquez.

Sánchez, C. (2014): Prácticas de lectura en el aula. Orientaciones didácticas para docentes, Bogotá, Ministerio de Educación / Cerlalc-Unesco.

Santos, B. (2006): Conocer desde el Sur. Para una cultura política emancipatoria, Lima, Fondo Editorial de la Facultad de Ciencias Sociales.

Smith, E. y S. Kosslyn (2008): Procesos cognitivos: modelos y bases neurales, Madrid, Pearson-Prentice Hall.

Universidad del Quindío (2011): Estrategia virtual. Una alternativa para los procesos de enseñanza aprendizaje, Armenia, Optigraf.

Vásquez, F. (2000): Oficio de maestro, Bogotá, Kimpres. 